the requirement for the PEXEL motif can be by-passed by the presence of a PNEP TM domain

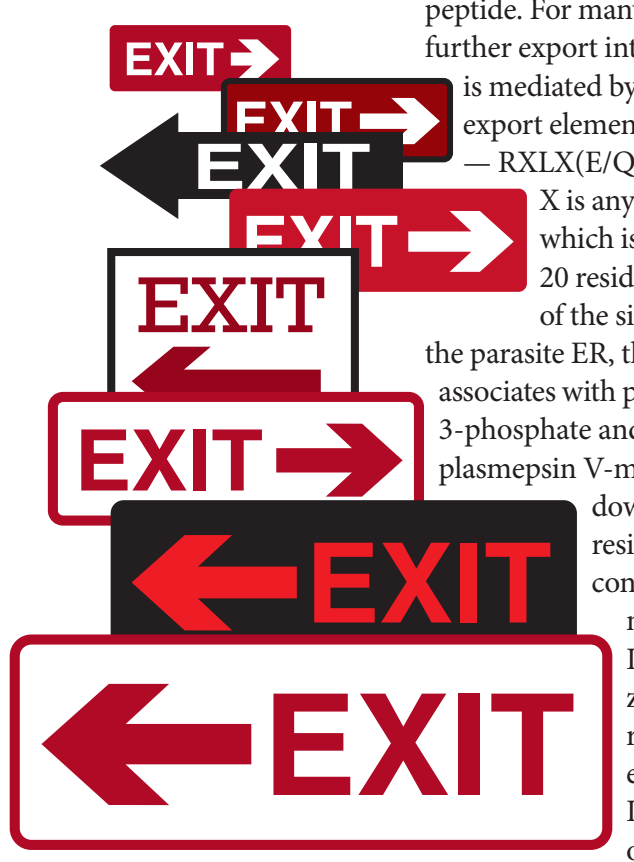

PARASITOLOGY

\title{
Layers of control for Plasmodium protein export
}

The export of effector proteins from Plasmodium falciparum into infected erythrocytes is crucial for the virulence of this apicomplexan parasite, as it facilitates the extensive remodelling of host cells that is necessary to create an effective replicative niche. Writing in Cell Host \& Microbe, Grüring and colleagues now reveal unexpected intricacies in the control of effector export.

$P$. falciparum effectors must cross two membranes, the parasite plasma membrane (PPM), and the parasitophorous-vacuole membrane (PVM) surrounding the vacuole in which the parasite resides. Entry into the secretory pathway of the parasite and transport to the parasitophorous vacuole is mediated by an amino-terminal signal peptide. For many effector proteins, further export into the host cell mediated by a Plasmodium export element (PEXEL) motif - RXLX(E/Q/D), in which $\mathrm{X}$ is any amino acid which is located roughly 20 residues downstream of the signal peptide. In the parasite ER, the PEXEL motif associates with phosphatidylinositol3-phosphate and undergoes plasmepsin V-mediated cleavage downstream of the Lys residue, leaving just one conserved residue at the mature $\mathrm{N}$ terminus. It was therefore puzzling how this single residue could confer export specificity. In addition, a group of PEXEL-negative

exported proteins (PNEPs) was identified; these proteins contain a hydrophobic region that can form a transmembrane (TM) domain, but do not contain a recognizable export motif. It had been suggested that the $\mathrm{N}$ termini of PNEPs resemble the mature, cleaved $\mathrm{N}$ termini of PEXEL proteins, meaning that there may be a common export pathway.

Grüring and colleagues began investigating this hypothesis using an export reporter, $\mathrm{R}^{\mathrm{REX} 2-\mathrm{TM}}$, based on a truncated version of the non-exported P. falciparum protein MTRAP fused to GFP and containing the TM region from the PNEP REX2. They found that this PNEP reporter was exported only when it contained a PNEP $\mathrm{N}$ terminus in addition to the TM region. To assess the requirements for PEXEL protein export, they added the first 20 residues of the mature $\mathrm{N}$ termini (which lacked most of the PEXEL motif) from two soluble PEXEL proteins and one TM PEXEL protein to $\mathrm{R}^{\mathrm{REX} 2-\mathrm{TM}}$. All the tested PEXEL protein $\mathrm{N}$ termini promoted export, suggesting that the requirement for the full PEXEL motif can be by-passed by the presence of a PNEP TM domain and confirming that the mature $\mathrm{N}$ terminus of a PEXEL protein is indeed equivalent to the $\mathrm{N}$ terminus of a PNEP.

Following cleavage of the PEXEL motif, a single conserved residue remains at what was position 5 , and there is a non-conserved residue at what was position 4 (giving XE, XQ or XD). As this combination could also be present in non-exported proteins, the authors speculated that perhaps additional information present in the mature $\mathrm{N}$ terminus allows discrimination between exported and non-exported proteins. The authors selected two non-exported proteins with mature $\mathrm{N}$ termini that start with the residues XE and $\mathrm{XQ}$ - and so resemble the processed PEXEL motif - and added the first 20 amino acids from these $\mathrm{N}$ termini to $\mathrm{R}^{\mathrm{REX} 2-\mathrm{TM}}$. These constructs were not exported, suggesting that the region of the mature $\mathrm{N}$ terminus downstream of the cleaved motif does confer discrimination. The authors confirmed this second level of export control in vivo by examining live P. falciparum expressing two reporter PEXEL proteins that differed only in the 20 residues following the PEXEL motif; even after correct PEXEL processing, export was not achieved in the presence of a downstream region that was refractory for export.

Last, the authors investigated the involvement of the putative translocon that has been identified at the PVM and is believed to be involved in the export of unfolded soluble PEXEL effectors into the erythrocyte. Using a modified unfolding assay, they found that inhibition of unfolding arrested the export of both TM PNEPs and soluble PEXEL proteins, suggesting that both types of protein undergo translocation. In an unexpected twist, however, rather than both groups being blocked for translocation across the PVM, it appeared that translocation of TM proteins was blocked at the PPM instead, suggesting that an additional trafficking step is involved in the export of these proteins.

Clearly, the intricacies of protein export in P. falciparum are only just beginning to be understood, and this study illustrates how much remains to be discovered about this complex process.

Sheilagh Molloy

ORIGINAL RESEARCH PAPER Grüring, C. et al. Uncovering common principles in protein export of malaria parasites. Cell Host Microbe 12, 717-729 (2012)

FURTHER READING Goldman, D. \& Cowman, A. Moving in and renovating: exporting proteins from Plasmodium into host erythrocytes. Nature Rev. Microbiol. 10, 617-621 (2010) 